\title{
Tangence
}

\section{Détections science-fictionnelles}

\section{Richard Saint-Gelais}

Numéro 38, décembre 1992

Fiction policière et roman actuel

URI : https://id.erudit.org/iderudit/025740ar

DOI : https://doi.org/10.7202/025740ar

Aller au sommaire du numéro

Éditeur(s)

Tangence

ISSN

1189-4563 (imprimé)

1710-0305 (numérique)

Découvrir la revue

Citer cet article

Saint-Gelais, R. (1992). Détections science-fictionnelles. Tangence, (38), 74-84.

https://doi.org/10.7202/025740ar d'utilisation que vous pouvez consulter en ligne.

https://apropos.erudit.org/fr/usagers/politique-dutilisation/ 


\section{Détections science-fictionnelles}

\section{Richard Saint-Gelais}

Qu'on me permette de commencer de façon bien banale, en remettant en question une idée reçue - une idée, qui plus est, qui n'est déjà plus si reçue que ça. À première vue, "roman policier. équivaut à: fiction construite autour d'une énigme à résoudre, et inversement. Les choses sont pourtant bien moins simples; le récit de détection n'est pas coextensif au genre policier. On sait par exemple que de nombreux romans policiers n'accordent pas un rôle prépondérant à la résolution de l'énigme, et que d'autres vont jusqu'à éliminer carrément cette dernière, en livrant immédiatement aux lecteurs des informations qui, traditionnellement, n'étaient révélées qu'à la toute fin du récit. Un exemple extrême, qu'on peut d'ailleurs lire comme parodique en raison de sa fulgurance, est offert par le $\hat{O}$ dingos, ô châteaux de Manchette, dont voici les toutes premières lignes:

L'homme que Thompson devait tuer, un pédéraste coupable d'avoir séduit le fils d'un industriel, entra dans la chambre. Refermant sa porte derrière lui, il eut le temps de sursauter à la vue de Thompson debout contre le mur à côté des gonds. Puis Thompson lui enfonça dans le cœur une lame de scie rigide $[\ldots]^{1}$

Le genre policier comprend donc, et depuis un bout de temps déjà, des récits qui ne sont pas des récits à énigme, des récits où l'attente de la suite remplace la perplexité à propos de ce qui a bien pu se passer. Mais ce n'est pas tout. On peut facilement montrer, réciproquement, que le modèle de la détection a été fréquemment repris en dehors du genre policier lui-même.

Cette migration peut s'expliquer, au moins en partie, par le caractère explicitement interactif du récit policier, qui ne peut pas être considéré simplement comme la narration d'une histoire, puisqu'il constitue un véritable dispositif de lecture. Plus précisément, un agencement discursif qui doit être pensé en fonction

1 Jean-Patrick Manchette, $\hat{O}$ dingos, ô châteaux, Paris, Gallimard, coll. - Série noire., 1972. 
des processus que les lecteurs mettent en œuvre face au texte: interrogations, inférences, hypothèses (que celles-ci soient justes ou non, d'ailleurs) et ainsi de suite. Cette façon de concevoir le texte comme un espace de lecture ne pouvait que séduire des écrivains (certains nouveaux romanciers par exemple) qui travaillent à problématiser les rapports entre texte et lecture. Aussi la reprise des structures policières s'est-elle accompagnée, chez ces écrivains, de transformations assez importantes par rapport au modèle classique du récit policier.

La plus évidente de ces transformations est sans doute le refus fréquent de résoudre l'énigme. Moins souvent notée, mais tout aussi importante, me paraît la façon dont l'énigme elle-même se constitue. Celle-ci, chez Robbe-Grillet ou Pinget par exemple, ne se pose plus uniquement en termes de données fictives (qui était où à quelle heure, et ainsi de suite), mais d'abord et avant tout en termes discursifs: c'est le texte lui-même qui suscite la perplexité du lecteur, et ce à cause de ses contradictions, de ses paradoxes, de ses aberrations, et ainsi de suite. Dans La maison de rendez-vous de Robbe-Grillet, lorsque la description d'une image se mue en description d'une scène animée, ou bien lorsque Édouard Manneret réapparaît comme si de rien n'était quelques dizaines de pages après avoir été assassiné, on admettra sans peine que les énigmes qui résultent de ces dispositifs ont fort peu à voir avec les énigmes d'Agatha Christie ou d'Ellery Queen. Le texte, dans de tels cas, n'est pas le compte rendu d'un problème qui résiderait ailleurs: il constitue lui-même un espace problématique, et on devine que dans ces conditions l'activité de lecture s'en trouve radicalement transformée.

Si cette reprise critique des structures policières par des écrivains modernes est un phénomène assez largement connu, moins étudiée, en revanche, est la réutilisation qui est faite de ces structures dans un tout autre cadre, celui de la science-fiction contemporaine. Il ne s'agit pourtant pas là d'un phénomène marginal puisque le dispositif de l'énigme joue un rôle central dans de nombreuses intrigues science-fictionnelles. Cependant, tout comme dans le cas de la littérature moderne, cette reprise ne va pas sans un certain nombre de transformations tenant au genre d'accueil. Ce sont ces avatars science-fictionnels du récit de détection que je voudrais examiner ici.

La première question qui se pose est: quelles sont les conséquences d'une hybridation de deux genres déjà bien constitués, 
comme le sont le roman policier et la science-fiction? Il ne s'agit pas de se demander à quel genre appartiendront les rejetons d'un tel croisement, mais plutôt de se demander quels types d'organisation du récit cela peut produire, quels rapports avec l'activité de lecture cela peut susciter.

On trouve une réponse implicite à la première question chez Uri Eisenzweig, lorsque celui-ci fait remarquer que la spécificité du récit policier est avant tout formelle. Eisenzweig ajoute que cela distingue le genre policier des autres genres paralittéraires (science-fiction, western, roman de cape et d'épée...), dont la spécificité serait, selon lui, plutôt thématique que structurelle ${ }^{2}$. Si l'on accepte cette hypothèse, on en concluera probablement que les tentatives d'hybridation entre roman policier et science-fiction produiront des récits où, formellement parlant, les structures policières prédomineront, dans la mesure où la science-fiction se réduirait à une quincaillerie thématique n'imposant aucune structure narrative particulière a priori. Le roman policier de sciencefiction apparaît alors comme une variété du genre policier, au même titre, par exemple, que les romans policiers médiévaux d'Ellis Peters ou d'Umberto Eco, les romans policiers de la Chine ancienne de Robert Van Gulik ou encore, plus près de nous, le roman policier inuit de Paul-René Bussière. L'exotisme de la planète Mars vaut bien celui de la dynastie des Ming ou du Grand Nord québécois, mais pas plus que ceux-là il n'impliquerait une modification structurelle du roman policier.

Si c'était le cas, je pourrais donc terminer mon investigation ici, à moins de me complaire dans les plaisirs équivoques de l'analyse thématique. Seulement, les choses, encore une fois, sont moins simples. On peut en effet imaginer l'objection suivante: du fait que les romans de science-fiction se déroulent dans des mondes fictifs qui sont considérablement différents de notre monde de référence - des mondes où des phénomènes comme les voyages intergalactiques, la télépathie, les déplacements temporels, etc., sont possibles - ils mettent à la disposition de leurs assassins tout un arsenal d'armes, et plus généralement de moyens que les lecteurs ne connaissent pas forcément. En termes moins intuitifs, on dira que la science-fiction se caractérise par la

2 Uri Eisenzweig, *Quand le policier devient genre , in Eisenzweig (éd.), Autopsies du roman policier, Paris, U. G. E., coll. *10/18*, 1983, p. 25-26. 
mise en place d'encyclopédies idiosyncrasiques (la formule est d'Umberto Eco ${ }^{3}$ ), c'est-à-dire de mondes de référence particuliers à chaque œuvre et que les lecteurs ont à reconstruire à mesure qu'ils progressent dans le texte. Or, dans le cas d'un récit de détection, ces différences idiosyncrasiques sont susceptibles d'avoir des conséquences sur les capacités des lecteurs à résoudre l'énigme. Pour une fois, il semble clair que les lecteurs et le détective ne sont pas sur le même pied: le second connaît les mille et une particularités du monde fictif, les premiers, non. L'hybridation entre roman policier et roman de science-fiction produirait donc non seulement des différences thématiques, mais aussi des différences qui affectent la pratique de lecture.

Le problème, on le voit, c'est que ces différences sont aussitôt perçues comme une menace vis-à-vis de l'intégrité du genre policier: comment préserver le fameux *fair-play * lorsque l'auteur peut sortir de sa manche, si je puis dire, un gadget qui résoud le mystère mais qui jusque-là était complètement inconnu des lecteurs? Cette question est examinée par Isaac Asimov dans l'introduction à son recueil Asimov's Mysteries ${ }^{4}$ - qui est un recueil de nouvelles policières de science-fiction. La réponse d'Asimov est simple. Il fait d'abord remarquer que les auteurs policiers peuvent tout aussi bien tricher, à leur façon, avec les lecteurs - mais qu'ils s'en abstiennent, précisément en leur donnant toutes les informations nécessaires. De la même façon, ajoute Asimov, les auteurs de récits policiers science-fictionnels n'ont qu'à prendre soin de fournir aux lecteurs toutes les données (je préciserais: les données encyclopédiques) sur lesquelles repose la solution de l'énigme.

Il n'est pas difficile de voir que la problématique adoptée par Asimov place ses récits dans la plus pure tradition du whodunit, où la tâche du détective (et des lecteurs) consiste à opérer les bonnes déductions, ou plutôt les bonnes abductions, à partir d'un ensemble d'indices. Par exemple, dans "The Dust of Death ", l'une des nouvelles du recueil, le coupable est un astronaute qui a séjourné plusieurs années sur Titan, dont l'atmosphère est constituée de méthane et d'hydrogène, et qui, une fois revenu sur Terre, décide d'assassiner son directeur de recherche en planifiant

3 Sémiotique et philosophie du langage, trad. fr. de Myriem Bouzaher, Paris, P. U. F., coll. . Formes sémiotiques *, 1988, p. 77.

4 Isaac Asimov, Asimov's Mysteries, New York, Doubleday \& Co, 1968. 
78

une explosion dans le laboratoire privé de ce dernier. Le coupable est démasqué parce qu'il a d'abord préparé l'explosion en fonction de l'atmosphère de Titan plutôt qu'en fonction de l'atmosphère terrestre, laissant ainsi des indices qui permettront au détective de remonter jusqu'à lui.

Les modifications par rapport au modèle classique semblent ici minimales: les éléments sur lesquels s'appuie le raisonnement du détective sont différents, certes, mais le modèle de la détection apparaît inchangé, y compris dans les chances qu'il laisse aux lecteurs de trouver eux aussi la solution. Mais, à y regarder de plus près, quelque chose est irrémédiablement modifié dans le rapport de la lecture et du récit. En effet, pour que le récit soit spécifiquement science-fictionnel, il faut que la solution implique des éléments encyclopédiques inédits par rapport au monde de référence des lecteurs - sans quoi les éléments sciencefictionnels ne serviraient qu'à poser un décor plus ou moins exotique mais sans incidence propre sur l'intrigue. Or ceci entraîne que les données encyclopédiques inédites deviennent particulièrement saillantes aux yeux des lecteurs: toute donnée qui n'appartient pas à notre monde de référence apparaît comme susceptible d'être reliée de près à la solution. Ceci devient manifeste après avoir lu quelques nouvelles. On peut donc parler d'un réglage progressif de la lecture, qui fait que les lecteurs sont de plus en plus avertis à mesure qu'ils avancent dans le recueil, dans la mesure bien entendu où ils tirent la leçon des énigmes précédentes. Je précise toutefois que ces remarques valent pour le recueil d'Asimov, mais qu'on ne saurait les généraliser à l'ensemble des récits policiers science-fictionnels: les Asimov's Mysteries ne représentent qu'une façon parmi d'autres d'hybrider roman policier et science-fiction.

Avant de considérer ces autres possibilités, je voudrais attirer l'attention sur une dernière particularité qu'on retrouve dans plusieurs des nouvelles d'Asimov. Les suspects sont souvent des personnages qui ont fait de longs séjours sur une autre planète avant de revenir sur Terre, où la plupart du temps le meurtre a lieu. C'est d'ailleurs ce qui fait échouer les plans de ces criminels, dont les agissements sur Terre sont déterminés et infléchis par ce qui est devenu au fil des mois leur monde de référence, qu'il s'agisse de Titan, de la Lune ou de Mercure. Ainsi, dans l'exemple que j'ai donné plus tôt, le coupable laisse un indice compromettant parce qu'il a planifié son crime comme s'il se trouvait sur 
Titan. On voit donc que la différence vraiment importante n'est pas celle qui prévaut entre le monde de référence du lecteur et le monde fictif construit par le texte; c'est plutôt l'écart entre les différents mondes de référence des personnages qui joue ici un rôle déterminant. Ceci contribue évidemment à simplifier la tâche du lecteur, qui est somme toute dans la même situation que l'enquêteur, plus précisément dans le même rapport d'altérité face aux mondes de référence des suspects. Mais ce n'est pas seulement une question de facilité ou de difficulté de la lecture. Cela implique surtout que les lecteurs peuvent mettre entre parenthèses la dimension discursive de leur activité, pour n'en considérer que les aspects cognitifs. Autrement dit, les lecteurs peuvent négliger qu'ils sont face à un texte, dans la mesure où ils sont amenés à s'identifier au détective, qui lui ne travaille pas à partir d'un texte mais bien à partir d'indices factuels; pour le détective, l'enquête se fait dans un monde bien réel et non pas dans un monde de papier. Le lecteur est ainsi amené à négliger sa propre situation de lecteur ${ }^{5}$.

En somme, les Asimov's Mysteries constituent une sorte de défi intergénérique, celui de préserver le modèle du récit de détection aussi intact que possible malgré la démultiplication des mondes de référence. Or, s'il ne faut pas généraliser l'expérience d'Asimov, c'est que d'autres écrivains utilisent les possibilités spécifiques de la science-fiction pour renouveler plus ou moins radicalement l'agencement même de l'intrigue policière. On pourrait citer de nombreux cas, mais je m'en tiendrai à deux exemples: The Door Into Summer de Robert Heinlein, "Le Fantôme du Kansas " de John Varley.

The Door Into Summer ${ }^{6}$, d'abord, n'est pas à proprement parler un récit de détection, mais contient un important épisode où le narrateur - ainsi que les lecteurs - sont placés devant une énigme à résoudre. En 1970, le narrateur, Dan Davis, est placé en

5 Il faut cependant noter que dans quatre des treize nouvelles l'enquêteur est l'extraterrologiste Wendell Urth, qui est un armcbair detective à la Dupin puisqu'il ne sort à peu près pas de sa maison, en raison de sa phobie des moyens de transport. Urth est donc un détective qui travaille essentiellement à partir des récits que lui fait l'inspecteur Davenport du Terrestrial Bureau of Investigation - d'où des effets métanarratifs qui ne sont pas entièrement négligeables.

6 Robert Heinlein, The Door Into Summer, New York, Signet Books, 1957 (1956). 
80

état d'hibernation par deux associés qui veulent prendre le contrôle de la petite entreprise que tous trois ont fondée ensemble; en hibernant Davis, ils parviennent ainsi à neutraliser celui-ci d'une manière tout à fait légale. Lorsque Davis sort de son *grand sommeil ", en l'an 2000, il découvre rapidement toute une série de faits intrigants. Qui est cette Mme Schultz, dont il n'a jamais entendu parler auparavant, et qui a essayé de communiquer avec lui depuis son réveil? Qui a volé le prototype de robot qu'il avait mis au point juste avant d'être placé en hibernation forcée? Comment se fait-il que des inventions ont été brevetées à son nom pendant son sommeil artificiel? Qui est derrière cela? Et finalement: qui est l'inconnu qui est parti avec son amie Ricky lorsque celle-ci est sortie à son tour de son hibernation?

Ce qui est frappant, à ce stade du roman, c'est l'écart entre la dimension encyclopédique du monde fictif, qui n'a rien de mystérieux, et la dimension diégétique, où surgissent toutes sortes de problèmes: le narrateur assimile (et nous communique) très vite les particularités du monde futur dans lequel il reprend conscience, mais toute une série de faits échappent à sa compréhension. Ceci peut donner l'impression que les liens entre le cadre science-fictionnel et l'intrigue policière sont minimaux. Au fond, l'élément science-fictionnel de cette histoire, la technique d'hibernation, jouerait le même rôle que l'absence de témoins lors d'un meurtre, dans un roman policier classique: elle permet qu'il y ait une énigme. Il en va de même lorsque le narrateur apprend que des expériences secrètes se font dans le domaine du voyage temporel: pour lui, ce qui se présente alors est la possibilité d'enquêter in proesentia en retournant dans le passé plutôt que de raisonner à partir d'indices.

Or l'habileté du roman d'Heinlein tient à ce qu'un élément science-fictionnel qui paraissait d'abord extérieur à l'intrigue permet de mettre en place une fausse piste: à un stade de l'enquête, les lecteurs sont amenés à croire que Ricky a été la victime d'un des nombreux escrocs qui profitent de l'état d'égarement dans lequel se trouvent ceux qui reviennent à la vie après une période d'hibernation. Puis, de façon beaucoup plus spectaculaire, c'est un élément science-fictionnel qui se révèle constituer à la fois l'énigme et la solution. Lorsque le narrateur retourne dans le passé (un passé qu'il n'a pas connu parce qu'il était en hibernation), il pose précisément les gestes dont les 
conséquences l'avaient tant intrigué dans le futur: c'est lui qui vole le prototype du robot (pour éviter que ses associés malhonnêtes ne mettent la main dessus), c'est lui qui fait breveter un appareil qu'il a découvert dans le futur, c'est lui, finalement, qui attend son amie Ricky lorsque sa période d'hibernation se termine. On a donc une intrigue où le même personnage est à la fois, mais sans qu'il le sache d'abord, le sujet et l'objet de l'enquête - d'où une intrigue qui n'est pas sans parentés, curieusement, à la fois avec celle d'CEdipe Roi et avec celle du Meurtre de Roger Ackroyd, où celui qu'on recherche n'est nul autre que le narrateur. Mais au delà de ces rapprochements, l'intérêt de The Door Into Summer réside dans la façon astucieuse dont l'intrigue exploite les possibilités propres à ce monde fictif, en l'occurrence l'hibernation et le voyage dans le temps. L'imbrication du policier et du science-fictionnel est donc beaucoup plus étroite qu'il pouvait sembler à première vue: si le début de l'histoire donnait l'impression d'un polar situé dans un cadre futuriste, la fin de l'intrigue montre que les événements énigmatiques étaient en fait des indices d'une donnée encyclopédique insoupçonnée: le voyage dans le temps.

En ce sens, on peut dire que ce roman de 1956 préfigure, à sa façon, une stratégie narrative qui était alors loin d'être répandue en science-fiction. Je m'explique. La stratégie narrative traditionnellement adoptée en science-fiction peut être qualifiée de "didactique : les textes mettent en place des mondes fictifs plus ou moins étranges en eux-mêmes, mais la présentation de ces mondes n'a rien d'étrange quant à elle, puisque les lecteurs sont dûment informés de toutes les particularités des mondes en question. Dans certains cas, qui ne sont pas les plus adroits, on peut facilement montrer que le texte pose implicitement un lecteur du milieu du vingtième siècle, et ce même si l'action est censée se passer, disons, en plein trente et unième siècle. Le conservatisme d'une certaine science-fiction n'est donc pas limité aux histoires qui s'y déroulent ou aux positions idéologiques qui y sont défendues: ce conservatisme est aussi, sinon d'abord, discursif.

On aurait cependant tort de réduire l'ensemble de la sciencefiction à ce modèle didactique. The Door Into Summer, par exemple, résiste déjà à ce modèle, puisque son intrigue repose en bonne partie sur le fait que des éléments encyclopédiques cruciaux ne sont pas donnés à lire immédiatement. Certes, cette 
retenue d'informations découle de la situation fictive du personnage: les lecteurs apprennent la possibilité du voyage temporel en même temps que Dan Davis. Un peu comme dans le cas des récits d'Asimov, on peut donc voir le personnage comme un intermédiaire qui partage l'ignorance du lecteur face au monde fictif, ce qui permet de justifier l'insertion progressive d'informations dans un texte qui, dès lors, n'a rien de problématique. En ce sens, on est tenté d'affirmer que le roman de science-fiction est plus une variante du récit de voyage (où l'instance narrative joue aussi ce rôle d'intermédiaire face à un monde inconnu) que du récit policier.

Mais ce n'est pas toujours le cas; dans d'autres récits sciencefictionnels, ce n'est pas seulement le monde fictif qui est énigmatique, mais aussi le texte lui-même. Lisons par exemple les premières lignes du "Fantôme du Kansas" de John Varley:

Ma banque est la Archimede Trust Association. C'est un établissement offrant toutes garanties de sécurité. Le personnel est courtois et ils ont leur propre service médical dont la seule fonction est d'établir les enregistrements qui sont ensuite stockés dans les chambres fortes. ${ }^{7}$

Ce qui est remarquable, ici, c'est que le texte, dans un premier temps, permet aux lecteurs de recourir - sans même s'en rendre compte, probablement - au cadre de référence qui leur est familier. Quelle banque qui n'offre pas toutes les garanties de sécurité? Dont le personnel n'est pas courtois avec les clients? Puis, sans crier gare, toutes sortes de problèmes surgissent: pourquoi diable une banque aurait-elle son propre service médical? Et quels sont ces enregistrements qui sont stockés dans les chambres fortes? Le plus troublant est que ces problèmes sont, si je puis dire, silencieux: ce ne sont pas ceux d'un personnage qui se réveille trente ans aprés avoir été congelé et qui découvre ébahi une société différente, c'est le problème d'un lecteur face à un texte qui mentionne ces éléments comme si de rien n'était, comme s'ils appartenaient au monde de référence du narrateur et n'avaient pas besoin, de son point de vue, d'être expliqués en long et en large. La suite est loin de clarifier les choses:

7 John Varley, "Le fantôme du Kansas ", in Dans le palais des rois martiens, Paris, Denoël, coll. *Présence du futur *), cité par Caroline Masseron et al. «Lire à l'école: analyses et propositions *, Pratiques, $\mathrm{n}^{\circ} 35$, octobre 1982, p. 49. 
Et, il y a deux semaines, ils ont été dévalisés.

Cela me donnait un répit. La date de mon contrôle de routine approchait et je songeais avec inquiétude à la ponction qu'il allait infliger à mes économies. ${ }^{8}$

Les énigmes, on le voit, se multiplient. En quoi le cambriolage de la banque pourrait-il donner un répit au narrateur ${ }^{9}$ ? Et quelle incidence le cambriolage a-t-il au juste sur le contrôle de routine? Quel contrôle de routine? Le lecteur se retrouve dans une situation embarrassante puisque chaque nouvelle information que lui donne le texte se paye au prix fort d'une incompréhension grandissante face aux institutions, aux façons de faire, aux attentes, etc., qui caractérisent ce monde fictif. Progresser dans le texte, c'est semble-t-il moins en apprendre davantage que se poser de plus en plus de questions. Où cela s'arrêtera-t-il ?

On notera en outre que les questions doivent être patiemment construites par le lecteur, à partir des présupposés du texte, ce qui les distingue des questions provoquées par exemple par le vocabulaire "extraterrestre " couramment utilisé dans une certaine science-fiction. Caroline Masseron montre bien cette différence lorqu'elle écrit:

Si je lis au début d'Oms en série [de Stefan Wull: "En silence, le draag s'approcha du hublot.... je sais que je ne sais pas ce qu'est un draag et je cherche l'information dans la suite, qui me l'apporte d'ailleurs vite et complaisamment. Ici [* La fantôme du Kansas.], aucun signal de ce genre: ce sont les mots de tous les jours qui vont jouer un rôle différent $[. . .]^{10}$

Le problème, on le voit, n'est pas que lexical; il s'agit surtout de distinguer, d'une part, une altérité que je dirais ostensible, qui s'affiche par des signes explicites dans un discours non problématique, et, d'autre part, une altérité plus discrète, plus troublante aussi parce que diffuse dans l'ensemble du texte. Et * Le fantôme du Kansas " est loin d'être un cas isolé: mériteraient d'être examinés, dans cette perspective, et parmi bien d'autres, certains textes de Stanislas Lem (en particulier Le rbume), des frères Strougatsky (Definitely Maybe), de Brian Aldiss (Report on Probability $A$ ), sans compter les romans science-fictionnels de

$8 \quad$ Ibid.

9 À la narratrice, en fait, mais cela aussi ne sera appris que plus loin.

10 Caroline Masseron, loc. cit., p. 50. 
84

Claude Ollier (La vie sur Epsilon) et de Jean Ricardou (La prise de Constantinople). Comme ces exemples le suggèrent, on atteint là une sorte de zone frontière entre la science-fiction, la littérature contemporaine et le récit de détection; une zone où les uns et les autres sont singulièrement transformés, et l'activité de lecture avec eux.

Il y a donc, au bout du compte, deux façons, au moins, de combiner science-fiction et détection. La première consiste, comme dans les Asimov's Mysteries et, dans une moindre mesure, dans The Door Into Summer, à insérer des éléments sciencefictionnels dans ce que j'appellerais une énigme restreinte, c'est-àdire une énigme qui se situe au niveau de l'intrigue: une énigme dont la solution apparaîtra assurément comme originale, que le lecteur pourra résoudre ou non selon sa perspicacité, mais une énigme qui est posée par un texte essentiellement non problématique. La seconde consiste à faire du texte tout entier un objet de détection. Le lecteur se trouve ainsi placé dans une situation où tous les éléments fictionnels sont susceptibles de relancer et de démultiplier les interrogations, constituant ainsi un dispositif où les énigmes ne sont plus circonscrites mais proliférantes. 